Bio - grafia. Escritos sobre la Biología y su Enseñanza. ISSN 2027

Edición Extraordinaria. p.p. 668 - 682

Memorias del VIII Encuentro Nacional de Experiencias en Enseñanza de la Biología y la Educación Ambiental. III Congreso Nacional de Investigación en Enseñanza de la Biología.

\title{
UNA MIRADA A LOS COMPORTAMIENTOS PROAMBIENTALES DE ESTUDIANTES DE GRADO NOVENO DE UN COLEGIO PÚBLICO DE BOGOTÁ D.C. EN EL CONTEXTO ESCOLAR
}

\author{
VIEW TO THE PROENVIRONMENTAL BEHAVIOURS BY THE NINTH GRADE \\ STUDENTS OF A PUBLIC SCHOOL IN BOGOTÁ D.C. IN THE CONTEXT SCHOOL
}

Barreto Tovar Carlos Humberto ${ }^{1}$ Martha Bibiana González Jiménez ${ }^{2}$

\section{RESUMEN}

El presente artículo expone los avances de investigación en relación con las dinámicas de construcción de una cultura ambiental en el aula de clase, basada en el estudio de las manifestaciones de los comportamientos proambientales de los estudiantes del grado noveno de educación básica secundaria, con miras a comprender desde una perspectiva integradora, algunos de los aspectos que inciden en la forma como los jóvenes se relacionan con su entorno biofísico y social, dentro del contexto escolar.

Desde ésta óptica se tuvieron en cuenta como elementos de diagnóstico, diferentes aspectos que influyen en la forma como ocurren los procesos de interrelación de los estudiantes con su entorno, desde los lineamientos y las políticas institucionales, hasta las condiciones que ofrece la planta física del colegio y la forma como se perciben desde los jóvenes las acciones a favor del ambiente en el contexto escolar.

\footnotetext{
${ }^{1}$ Universidad de la Sabana. Facultad de Educación. Maestría en Educación. Docente - Investigador. Correo: carloshumbertobarreto@hotmail.com

2 Universidad de la Sabana. Facultad de Educación. Maestría en Pedagogía. Coinvestigadora. Docente Colegio Costa Rica IED. Sede A. Jornada Mañana. Correo: marthagoji@unisabana.edu.co
} 
Bio - grafia. Escritos sobre la Biología y su Enseñanza. ISSN 2027

Edición Extraordinaria. p.p. 668 - 682

Memorias del VIII Encuentro Nacional de Experiencias en Enseñanza de la Biología y la Educación Ambiental. III Congreso Nacional de Investigación en Enseñanza de la Biología.

Este estudio, constituye la base para diseñar posteriormente estrategias pedagógicas que permitan que los estudiantes, de una manera consciente, deliberada y autónoma, transformen sus prácticas culturales hacia estilos de vida más sustentables, mediante la promoción de comportamientos proambientales que puedan luego instaurarse en contextos más amplios a nivel institucional, familiar, local y global.

PALABRAS CLAVE: Educación Ambiental, conocimientos ambientales, actitudes proambientales, comportamientos proambientales, cultura ambiental, Proyecto Ambiental Escolar (PRAE).

\section{ABSTRACT}

This article shows the investigation advances in relation with the dynamics of construction of an environmental culture in the classroom, based in the study of proenvironmental manifestations of ninth grade students, in basic school education, with the aim of understanding from a integrating perspective, some of the aspects that influence in the way youngsters related their biophysical and social surrounding, inside the school context.

From this view, it was considered as diagnostic elements different aspects that influence the way how the students' interrelation with their surroundings happen, from the institutional guidelines and policies, to the conditions offered by the school facilities and the way how youngsters perceive the actions in favour of the environment in the school.

This research, constitutes the base for a posterior design of pedagogical strategies that allow the promotion of pro-environmental behaviours in the students, so, in a consistent, deliberated, and autonomous way they transform their cultural practices to more sustainable life styles and which could later be applied in wider contexts at institutional, family, local and global levels.

Key Words: environmental education, pro-environmental behaviour, environmental culture 
Bio - grafia. Escritos sobre la Biología y su Enseñanza. ISSN 2027

Edición Extraordinaria. p.p. 668 - 682

Memorias del VIII Encuentro Nacional de Experiencias en Enseñanza de la Biología y la Educación Ambiental. III Congreso Nacional de Investigación en Enseñanza de la Biología.

\section{INTRODUCCIÓN}

Este artículo muestra avances de la investigación que viene desarrollándose en el marco de la Maestría en Pedagogía de la Universidad de la Sabana, titulada "Implementación de estrategias pedagógicas para la promoción de comportamientos proambientales en estudiantes de noveno grado de básica secundaria en el colegio Costa Rica IED".

Para ello, se tomaron algunos elementos provenientes de la Psicología Ambiental para trabajarlos en la escuela, disciplina que constituye un referente fundamental en la comprensión de las interrelaciones entre las personas, el ambiente y los espacios sociofísicos, para lograr un acercamiento desde la percepción individual y colectiva sobre el ambiente (Moser, 2014). Autores como Bayón (2006), citado por Miranda (2013), definen la cultura ambiental, como "La forma en la que los seres humanos se relacionan con el medio ambiente, incluyendo el conjunto de estilos, costumbres y condiciones de vida de una sociedad con una identidad propia, basada en tradiciones, valores y conocimientos". Con base en ésta definición, la escuela como espacio de interacción social, posee unas dinámicas únicas y complejas frente a los procesos de construcción de la cultura ambiental que han sido hasta hoy, poco estudiadas.

Dada la amplitud del concepto de cultura ambiental, éste estudio se enfoca en los aspectos referentes a las actitudes, entendidas como los sentimientos favorables o desfavorables que se tienen hacia alguna característica del medio, o como una predisposición aprendida para actuar en forma favorable o desfavorable con respecto a la elección de un objeto; de acuerdo con esto, las actitudes pueden ser abordadas desde tres elementos: cognitivos, afectivos y conductuales (Fishbein y Azjen, 1975, citado por Páramo y Gómez, 1997). En este sentido, una visión de la Educación Ambiental desde la perspectiva de de las actitudes y los comportamientos proambientales, permite ir más allá de un enfoque reduccionista del ambiente, que se limita a la inclusión de contenidos ecológicos dentro del currículo, para dar relevancia, visibilizar y movilizar emociones, comportamientos, conocimientos y competencias proambientales vinculadas a acciones concretas sobre el cuidado del entorno. 
Bio - grafia. Escritos sobre la Biología y su Enseñanza. ISSN 2027

Edición Extraordinaria. p.p. 668 - 682

Memorias del VIII Encuentro Nacional de Experiencias en Enseñanza de la Biología y la Educación Ambiental. III Congreso Nacional de Investigación en Enseñanza de la Biología.

Para profundizar en este campo, Corral (2001), citado por Corral (2010), define los comportamientos proambientales, o conductas proecológicas como "el conjunto de acciones deliberadas y efectivas que responden a requerimientos sociales $e$ individuales y que resultan en la protección del medio". En lo que respecta a la relación entre conocimientos y comportamientos proambientales, Mata et al, (2002), citado por Mata (2004), señala que los conocimientos en materia ambiental son uno de los precursores de las creencias que influyen sobre las conductas, pero no constituyen un buen predictor, ya que no son suficientes por sí mismos, aclarando que cuando involucran otras dimensiones del ser humano, como lo afectivo, lo ético y lo conductual, pueden constituirse en una adecuada puerta de entrada para la transformación de la cultura ambiental. En la misma línea, Mera (2003), indica que a pesar de abordarse la temática ambiental en las instituciones, ésta información no se ha convertido en conocimiento ya que no se ha interiorizado ni ligado a una red conceptual con sentido y significado.

A partir de estas definiciones, cabe preguntarnos en términos pedagógicos, sobre las estrategias de enseñanza y aprendizaje más adecuadas para lograr la construcción de conductas pro ambientales, desde la escuela. En éste sentido, el trabajo planteado constituye un aporte para abordar la Educación Ambiental desde una visión más amplia que involucre las emociones, conductas y conocimientos necesarios para desarrollar competencias proambientales en los estudiantes, apoyándose en una pedagogía basada en la evidencia que conduzca a investigar la realidad educativa a partir de lo que muestra la experiencia, la observación cuidadosa, la demostración de hipótesis, la recolección sistemática de información a las preguntas de investigación y la acción pedagógica por los resultados que produce, poniendo a prueba los planteamientos teóricos en situaciones prácticas (Páramo, 2014). Es así como se espera que al intervenir pedagógicamente sobre las actitudes manifestadas por los estudiantes hacia el ambiente, se logre promover comportamientos proambientales que permitan evidenciar un impacto positivo frente al cuidado del entorno, inicialmente en el contexto escolar. 
Bio - grafia. Escritos sobre la Biología y su Enseñanza. ISSN 2027

Edición Extraordinaria. p.p. 668 - 682

Memorias del VIII Encuentro Nacional de Experiencias en Enseñanza de la Biología y la Educación Ambiental. III Congreso Nacional de Investigación en Enseñanza de la Biología.

\section{METODOLOGÍA}

El estudio se lleva a cabo en la Institución Educativa Distrital Colegio Costa Rica, ubicada en la localidad de Fontibón, la cual cuenta con tres sedes y tres jornadas, para la atención en los diferentes niveles educativos, desde la primera infancia, hasta la media vocacional. La institución cuenta con un total de 1446 estudiantes, 128 docentes, 8 administrativos, 7 directivos docentes y 16 personas de servicios generales, para la prestación del servicio educativo. El colegio está ubicado en un sector mayoritariamente industrial y comercial; los estudiantes corresponden a los estratos dos y tres, y provienen en gran medida de la misma localidad. La población de estudio corresponde a cinco grupos del grado noveno, que al iniciar el año escolar 2015 suman en total 180 estudiantes.

Este estudio de carácter cualitativo es, inicialmente, de tipo exploratorio (Sampieri, 1991), ya que una vez revisada la literatura alrededor de investigaciones relacionadas con las actitudes y los comportamientos proambientales, se observa una tendencia hacia el trabajo con poblaciones adultas. En el campo de la educación formal los estudios encontrados se han llevado a cabo, principalmente a nivel universitario, por lo cual éste trabajo aportaría en la consolidación de una literatura científica frente al tema, en los niveles de la básica secundaria, donde no ha sido suficientemente explorado, permitiendo además abrir nuevas posibilidades de investigación en torno a la forma de abordar desde la pedagogía, las dimensiones de la cultura ambiental escolar.

La primera fase del trabajo comprende un diagnóstico sobre las características del contexto escolar estudiado, con base en documentos institucionales, observación directa de las condiciones de la planta física y las dinámicas propias del aula que evidencian actitudes y comportamientos ambientales de los estudiantes. Se llevó a cabo a través de las siguientes estrategias: 
Bio - grafia. Escritos sobre la Biología y su Enseñanza. ISSN 2027

Edición Extraordinaria. p.p. 668 - 682

Memorias del VIII Encuentro Nacional de Experiencias en Enseñanza de la Biología y la Educación Ambiental. III Congreso Nacional de Investigación en Enseñanza de la Biología.

- Recopilación y revisión de documentos institucionales como el PEI, el documento PRAE, la matriz de diagnóstico del PRAE propuesta por la Secretaría de Educación Distrital, la evaluación tipo DOFA del PRAE y los planes de estudio del área de Ciencias Naturales y Educación Ambiental. A la luz de estos documentos se pretende analizar la interrelación, el nivel de coherencia y el logro de las metas propuestas en materia de Educación Ambiental.

- Caracterización del aula de clase: se realizaron durante dos semanas mediciones de la temperatura ambiente en tres momentos de la jornada, al igual que la intensidad sonora a través de la aplicación Sound Meter, un sonómetro descargado de la web e instalado en un dispositivo móvil tipo android. Así mismo se observaron características en cuanto a área, luminosidad, tipo y estado del mobiliario, materiales de construcción. Todo esto con el fin de recopilar información sobre los factores ambientales y físicos del entorno, que permitan establecer la forma como éste puede influir en la manifestación de ciertas actitudes y comportamientos a favor o en contra del ambiente. Éste tipo de información puede orientar al docente para optimizar sus prácticas educativas, en términos de adaptar y aprovechar mejor los espacios y las condiciones que ofrece el aula para diferentes momentos del aprendizaje.

- Observación participante: llevada a cabo desde el inicio del año escolar 2015. Con la información recolectada a partir de los diarios de campo, realizados durante las clases de biología en cinco grupos del grado noveno del colegio Costa Rica IED, en diferentes escenarios de aprendizaje, se pretende dar cuenta de las dinámicas que ocurren al interior del aula, en cuanto a las actitudes y comportamientos ambientales de los estudiantes.

- Aplicación de un cuestionario con pregunta abierta: se entregó a un grupo de 50 estudiantes del grado noveno, una matriz con la pregunta: ¿Durante el último mes, qué acciones para cuidar el ambiente has realizado y cuáles has observado que realizan tus compañeros y profesores en el colegio? Éste subgrupo estuvo conformado por 24 hombres y 26 mujeres, con edades entre los 13 y 18 años. El instrumento permitió 
Bio - grafia. Escritos sobre la Biología y su Enseñanza. ISSN 2027

Edición Extraordinaria. p.p. 668 - 682

Memorias del VIII Encuentro Nacional de Experiencias en Enseñanza de la Biología y la Educación Ambiental. III Congreso Nacional de Investigación en Enseñanza de la Biología.

recopilar información preliminar sobre los comportamientos proambientales observados por los estudiantes en el entorno escolar.

\section{RESULTADOS Y DISCUSIÓN}

\section{Revisión de documentos Institucionales}

Los resultados arrojan un trabajo teórico interrelacionado, en cuanto a los principios y objetivos propuestos. EI PEI de la institución gira en torno a la "Formación de líderes con responsabilidad social", y aunque se encuentra en construcción, la misión y visión apuntan al desarrollo de las competencias académicas y los valores necesarios para generar procesos de liderazgo y transformación social (Costa Rica IED, 2014). Los documentos relacionados con el planteamiento, ejecución, diagnóstico y evaluación del PRAE, muestran como debilidades la falta de interdisciplinariedad, la baja participación de todos los estamentos de la comunidad educativa y la apatía frente al tema, aspectos que ya han sido reportados en otros estudios sobre el impacto de los PRAE en las comunidades educativas (Herrera, Reyes, Amaya \& Gerena, 2006). Cabe resaltar que las estrategias contempladas en el PRAE, han fortalecido en los últimos años aspectos relacionados con el manejo de residuos, el ornato y embellecimiento de la planta física. Sin embargo, se requiere involucrar a toda la comunidad educativa, ya que estas acciones son llevadas a cabo principalmente por el comité ambiental escolar.

En éste sentido, se hace necesario generar procesos de investigación que permitan un mayor acercamiento a la realidad ambiental de la institución, con el fin de fortalecer los espacios de construcción y participación de la comunidad educativa en acciones tendientes a mejorar la forma como los individuos se relacionan con su entorno biofísico y social. 
Bio - grafia. Escritos sobre la Biología y su Enseñanza. ISSN 2027

Edición Extraordinaria. p.p. 668 - 682

Memorias del VIII Encuentro Nacional de Experiencias en Enseñanza de la Biología y la Educación Ambiental. III Congreso Nacional de Investigación en Enseñanza de la Biología.

Caracterización de los espacios físicos del aula de clase

Las observaciones y mediciones realizadas al interior del aula y registradas a través de los diarios de campo pueden verse en la siguiente tabla:

Tabla 1. Características físicas del aula de clase 305 A- IED Costa Rica

\begin{tabular}{cc}
\hline Característica & \\
\hline Número promedio de estudiantes por curso & 36 \\
\hline Área total & $53,5 \mathrm{~m}^{2}$ \\
\hline Área por estudiante & $1,48 \mathrm{~m}^{2}$ \\
\hline Luminosidad & Excelente \\
\hline Promedio de Temperatura al iniciar la jornada & $17.7^{\circ} \mathrm{C}$ \\
\hline Promedio de Temperatura al finalizar la jornada & $24^{\circ} \mathrm{C}$ \\
\hline Promedio de decibeles en horas de clase & $82 \mathrm{~dB}$ \\
\hline Promedio de decibeles sin estudiantes & $74 \mathrm{~dB}$ \\
\hline
\end{tabular}

De acuerdo con la bibliografía, las condiciones físicas del espacio, pueden afectar el comportamiento, la disposición del grupo y la manifestación de conductas proambientales. Tal y como señala Moser (2014), factores relacionados con un aumento en la temperatura pueden generar comportamientos como irritación, agresión, cansancio y reacciones evasivas, lo que se podría traducir en el aula como apatía, desmotivación, aumento de conflictos interpersonales y disminución de la atención. En este punto, tanto los docentes, como los mismos estudiantes, pueden desarrollar actividades de observación y registro de estos factores, de tal forma que logren reconocer los problemas ambientales que se generan en el aula, y así mismo plantear alternativas de solución.

En el caso del colegio Costa Rica IED, la afectación más importante en términos de los factores físicos que interfieren en la disposición de los estudiantes para el aprendizaje y el ambiente de clase, es la exposición al ruido, generada por el tráfico vehicular y aéreo, dada la cercanía de una avenida principal y del aeropuerto El Dorado. 
Bio - grafia. Escritos sobre la Biología y su Enseñanza. ISSN 2027

Edición Extraordinaria. p.p. 668 - 682

Memorias del VIII Encuentro Nacional de Experiencias en Enseñanza de la Biología y la Educación Ambiental. III Congreso Nacional de Investigación en Enseñanza de la Biología.

La medición de la presión sonora $(\mathrm{dB})$, en ausencia de estudiantes corresponde a $74 \mathrm{~dB}$ y en horas clase es de $82 \mathrm{~dB}$ en promedio, como se observa en la Tabla 1, superando los niveles establecidos para el subsector de universidades, escuelas y centros de estudio, que de acuerdo a la norma no deben sobrepasar en horario diurno un máximo de $65 \mathrm{~dB}$, según la resolución de emisión de ruido y ruido ambiental 0627(2006). Para la OMS, un nivel de ruido que supere los $65 \mathrm{~dB}$ empieza a ser nocivo para la salud humana (Observatorio Salud y Medio Ambiente, 2012).

En cuanto a la densidad, entendida como el número de personas que ocupan una superficie determinada y relacionada a su vez con el espacio personal (Moser, 2014), constituye un factor preocupante, dado que el cálculo obtenido de $1,48 \mathrm{~m}^{2}$ por estudiante (Ver Tabla 1), no se ajusta al parámetro adecuado según las normas Icontec, para el Planeamiento y Diseño de Instalaciones y Ambientes Escolares, la cual dispone como indicador $1,80 \mathrm{~m}^{2}$, en el caso de espacios de aula para básica secundaria, con mobiliario unipersonal (MEN, 2006). Por lo anterior, se puede deducir que éste factor podría también generar situaciones de estrés, agresión o reacciones hostiles entre nuestros estudiantes.

\section{Comportamientos proambientales}

Constituyen una de las manifestaciones más evidentes para la medición de las actitudes ambientales, junto con los aspectos cognitivos y emocionales que los influencian (Páramo y Gómez, 1997). A través de un cuestionario, los estudiantes expresaron haber realizado y observado durante el último mes, algunos comportamientos proambientales en el ámbito escolar, los cuales han sido categorizados según Corral (2010). En las tablas 2 y 3 se han incluido los comportamientos proambientales relacionados con el contexto escolar, desde una mirada de los propios estudiantes.

Los estudiantes manifiestan en mayor medida que llevan a cabo acciones proambientales relacionadas con la estética y el ahorro de agua. 
Bio - grafia. Escritos sobre la Biología y su Enseñanza. ISSN 2027

Edición Extraordinaria. p.p. 668 - 682

Memorias del VIII Encuentro Nacional de Experiencias en Enseñanza de la Biología y la Educación Ambiental. III Congreso Nacional de Investigación en Enseñanza de la Biología.

Los aspectos relacionados con la estética reportados, tienen que ver en gran medida con conservar limpios los sitios donde interactúan, más no con la disminución del consumo o la generación de desechos (Corral, 2010). Se observa una confusión entre las acciones que tienen que ver con el reciclaje y el reuso de materiales de desecho, ya que para ellos el hecho reutilizar un objeto, ya constituye una acción de reciclaje. En éste sentido, es evidente que falta abordar la diferencia en términos de optimización de recursos y ahorro energético de los dos procesos, para continuar incentivando en mayores proporciones la reducción y el reuso de los materiales (Corral, 2010).

Tabla 2. Comportamiento de los estudiantes en el entorno escolar

\begin{tabular}{|c|c|c|c|}
\hline Categoría & $\begin{array}{l}\text { Comportamiento proambiental } \\
\text { escolar (estudiantes) }\end{array}$ & Frecuencia & $\%$ \\
\hline Acciones de Estética & Sembrar plantas & 14 & $40,4 \%$ \\
\hline \multirow[t]{6}{*}{ ambiental } & Recoger la basura & 8 & \\
\hline & Usar las canecas & 7 & \\
\hline & Mantener el salón limpio & 4 & \\
\hline & No botar basura & 3 & \\
\hline & No rayar los puestos & 1 & \\
\hline & Decorar los salones & 1 & \\
\hline \multirow[t]{4}{*}{ Reciclaje } & Recolectar tapas plásticas & 11 & $26,5 \%$ \\
\hline & Reciclar & 10 & \\
\hline & Reciclar las bolsas del refrigerio & 4 & \\
\hline & No sabe/ no responde & 10 & $10,6 \%$ \\
\hline \multirow[t]{3}{*}{ Cuidado de ecosistemas } & Regar las plantas & 1 & \\
\hline & Cuidar las plantas & 5 & $6,3 \%$ \\
\hline & Ninguno & 6 & $6,3 \%$ \\
\hline \multirow[t]{2}{*}{ Reuso de desechos } & Usar el papel por ambas caras & 1 & $5,3 \%$ \\
\hline & Reutilizar materiales & 4 & \\
\hline Ahorro de agua & No desperdiciar agua & 4 & $4,2 \%$ \\
\hline
\end{tabular}


Bio - grafia. Escritos sobre la Biología y su Enseñanza. ISSN 2027

Edición Extraordinaria. p.p. 668 - 682

Memorias del VIII Encuentro Nacional de Experiencias en Enseñanza de la Biología y la Educación Ambiental. III Congreso Nacional de Investigación en Enseñanza de la Biología.

En el contexto escolar, los estudiantes manifestaron, como lo muestra la Tabla 2, mayor inclinación a ejecutar acciones proambientales relacionadas con la estética, ya que el tema del embellecimiento y ornato constituye una de las prioridades de ejecución del PRAE.

Cabe anotar que los comportamientos proambientales manifestados por los estudiantes, se suscriben dentro de una concepción reduccionista del ambiente, donde sólo se tiene en cuenta los elementos biofísicos, excluyendo los aspectos sociales que abarcan las formas de interrelación con el otro; esto puede deberse a la falta de consenso entre lo que se plantea dentro del currículo para la enseñanza de la Educación Ambiental en la escuela y las necesidades actuales en materia ambiental (Benegas y Marcén, 1995).

Tabla 3. Comportamientos proambientales de los docentes en el contexto escolar

\begin{tabular}{cccc}
\hline Categoría & $\begin{array}{c}\text { Comportamiento proambiental escolar } \\
\text { (docentes) }\end{array}$ & Frecuencia & $\%$ \\
\hline Acciones de & Siembran plantas y nos enseñan a sembrarlas & 14 & $46,1 \%$ \\
estética ambiental & Recogen las bolsas del refrigerio & 10 & \\
& Recogen la basura & 6 & \\
& No botan basura al piso & 6 & \\
& Exigen limpieza y cuidado de los salones & 6 & \\
& Tienen plantas en los salones & 4 & \\
& Hacen la decoración sobre la naturaleza & 2 & $14,4 \%$ \\
Persuasión & Nos enseñan la importancia de cuidar el & 8 & \\
proecológica & medio ambiente & & \\
& Fomentan la reutilización de materiales & 4 & \\
& reciclables & 2 & \\
& Nos enseñan a reciclar & 1 & $11,5 \%$ \\
Reciclaje & Aconsejan a los estudiantes & 8 & \\
& Reciclan & 3 & \\
& Recogen las tapas plásticas & 1 & \\
& Traen tapas plásticas &
\end{tabular}


Bio - grafia. Escritos sobre la Biología y su Enseñanza. ISSN 2027

Edición Extraordinaria. p.p. 668 - 682

Memorias del VIII Encuentro Nacional de Experiencias en Enseñanza de la Biología y la Educación Ambiental. III Congreso Nacional de Investigación en Enseñanza de la Biología.

\begin{tabular}{cccc}
\hline Reuso de desechos & Reutilizan las hojas & 5 & $16,3 \%$ \\
& $\begin{array}{c}\text { Reutilizan materiales } \\
\text { Hacen manualidades con material reciclable } \\
\text { Asignan trabajos con materiales reciclables }\end{array}$ & 3 & \\
\hline $\begin{array}{c}\text { Reducción en el } \\
\text { consumo de } \\
\text { productos }\end{array}$ & Envían los trabajos por internet & 3 & $2,8 \%$ \\
\hline Ahorro de agua & & & \\
\hline & (no se especifica) & 6 & $5,7 \%$ \\
\hline
\end{tabular}

*Datos obtenidos a partir de los estudiantes

En cuanto a la forma como son percibidos los comportamientos proambientales de los maestros, registrados por los estudiantes, se evidencia en la Tabla 3, un alto impacto de las acciones relacionadas con la estética, enmarcadas en el desarrollo de actividades promovidas principalmente por el área de ciencias naturales. En menor proporción, los estudiantes han observado otros comportamientos ambientales que han generado impacto positivo en ellos, por ejemplo el reuso de desechos y la persuasión ecológica. En éste sentido, empieza a verse la Educación Ambiental como un proceso de desarrollo personal, antes que una práctica generadora de conocimientos, tanto para los alumnos como para los profesores, como lo manifiestan Benegas y Marcén (1995).

Una vez finalizada la etapa diagnóstica, se procederá a diseñar, implementar y evaluar algunas estrategias pedagógicas para la promoción de comportamientos proambientales, desde el aula de clase. Para ello, tal y como señalan Benegas y Marcén (1995), es necesario tener en cuenta aspectos como el modelo educativo, las características ambientales, los valores del grupo social, las características, inquietudes y estilos de aprendizaje del alumnado y las características e inquietudes de los docentes, con el fin de que la propuesta genere elementos innovadores que impacten y transformen las prácticas educativas en materia ambiental en los contextos escolares. 
Bio - grafia. Escritos sobre la Biología y su Enseñanza. ISSN 2027

Edición Extraordinaria. p.p. 668 - 682

Memorias del VIII Encuentro Nacional de Experiencias en Enseñanza de la Biología y la Educación Ambiental. III Congreso Nacional de Investigación en Enseñanza de la Biología.

\section{CONCLUSIONES}

Los resultados obtenidos hasta el momento permiten evidenciar la necesidad de implementar estrategias pedagógicas para la promoción de comportamientos ambientales, a partir de la comprensión de las dinámicas del aula, teniendo en cuenta todos los factores que intervienen en la interrelación de los estudiantes con el ambiente biofísico y social. Así mismo, ésta propuesta deberá responder frente al cómo interiorizar las conductas proambientales de tal forma que se conviertan en un estilo de vida.

Es necesario profundizar acerca de las complejas relaciones entre las actitudes y comportamientos proambientales, para esclarecer si lo que manifiestan los estudiantes corresponde a comportamientos reales o a "intenciones de comportamiento", asociadas a la responsabilidad que generan las normatividades (Moser, 2014). En el aspecto relacionado con los conocimientos ambientales, es necesario que las estrategias a implementarse permitan aclarar conceptos frente al reciclaje y la reutilización de desechos, ya que es evidente que hay confusión entre éstos dos procesos. Finalmente, aunque no es el objetivo de ésta investigación, la lectura que hacen los estudiantes sobre los comportamientos proambientales de los docentes ofrece valiosa información sobre las dinámicas de construcción de la cultura ambiental desde el aula de clase, tales como la enseñanza, a través del ejemplo, de valores y actitudes en torno al cuidado del entorno, el compromiso interdisciplinario para abordar los temas ambientales y el consenso en los fines educativos institucionales. 
Bio - grafia. Escritos sobre la Biología y su Enseñanza. ISSN 2027

Edición Extraordinaria. p.p. 668 - 682

Memorias del VIII Encuentro Nacional de Experiencias en Enseñanza de la Biología y la Educación Ambiental. III Congreso Nacional de Investigación en Enseñanza de la Biología.

\section{REFERENCIAS}

- Benegas, J. y Marcén, C. (1995). La Educación Ambiental como desencadenante del cambio de actitudes ambientales. Revista Complutense de Educación. Vol. 6. Pp. 1128

- Corral, V. (2010) Psicología de la sustentabilidad. Un análisis de lo que nos hace pro ecológicos y pro sociales. Ed. Trillas. México.

- Costa Rica IED (2014). Manual de convivencia.

- Herrera, J., Reyes, L., Amaya, H., \& Gerena, O. (2006). Evaluación de los Proyectos Ambientales Escolares en colegios oficiales de la localidad 18 en Bogotá. Obtenido de. 〈http://www.redalyc.org/articulo.oa?id=169421183009> ISSN 0124-177X . Revisado el 25 de mayo de 2014.

- Mata, A. (2004). Transformación de la cultura ambiental mediante la docencia universitaria. Biocenosis. Vol. 18 (1-2), 131.

- Mera, A. (2003). Apuntes para una reflexión tendiente al fortalecimiento de una cultura ambiental en Colombia. Umbral Científico. Universidad Manuela Beltrán. Nún.2, junio.

- Miranda, L. (2013). Cultura ambiental: un estudio desde las dimensiones de valor, creencias, actitudes y comportamientos ambientales. Producción más limpia, 94-105.

- Ministerio de Educación Nacional (2006). Normas Técnicas Colombianas NTC 4595. Disponible en http://www.mineducacion.gov.co/1621/articles96894_Archivo_pdf.pdf. Revisado el 31 de abril de 2015.

- Moser, G. (2014). Psicología Ambiental. Aspecto de las relaciones individuomedioambiente. Ecoe Ediciones. Bogotá.

- Observatorio Salud y medio ambiente (2012). Ruido y salud. Núm. 03. Pp. 26. España. Disponible en http://www.ecodes.org/noticias/el-informe-ruido-y-salud-sepresenta-con-motivo-del-dia-mundial-contra-el-ruido\#.VUP9vHRZgcA. Revisado el 31 de marzo de 2015. 
Bio - grafia. Escritos sobre la Biología y su Enseñanza. ISSN 2027

Edición Extraordinaria. p.p. 668 - 682

Memorias del VIII Encuentro Nacional de Experiencias en Enseñanza de la Biología y la Educación Ambiental. III Congreso Nacional de Investigación en Enseñanza de la Biología.

- Páramo, P. y Gómez, F. (1997). Actitudes hacia el medio ambiente: Su medición a partir de la teoría de facetas. Bogotá.

- Páramo, P. (2014). Educación basada en la evidencia. Revista Colombiana de Educación. No. $66 . \quad$ Bogotá. Disponible en: http://www.scielo.org.co/scielo.php?pid=S012039162014000100001\&script=sci_arttext

- Resolución 0627 (2006). Ministerio de Salud. Disponible en http://www.alcaldiabogota.gov.co/sisjur/normas/Norma1.jsp?i=19982. Revisado el 30 de abril de 2015.

- Sampieri, R., Fernández, C., \& Baptista, P. (1991). Metodología de la Investigación. México: Mc GRAW-H 